\title{
Entwicklung angepasster \\ Konstruktionsmethoden für \\ nachhaltige Hochvolt-Speicher
}

Robert Kretschmann, Gerd Wagenhaus, Christiane Beyer

Der vorliegende Posterbeitrag beschäftigt sich mit der Entwicklung angepasster Entwicklungsmethoden für nachhaltig aufgebaute Hochvolt-Speicher. Dazu wird zunächst analysiert, welche technischen Kriterien bei der Produktgestaltung vor dem Hintergrund der Wiederverwendbarkeit einzelner Komponenten besondere Berücksichtigung finden müssen. Unter Zuhilfenahme der Recycling-Kaskade aus der VDI 2243 wird die Entscheidungsfindung im Konstruktionsprozess methodisch ergänzt sowie die notwendigen Betrachtungen, die das Vorgehen objektiv bewerten, vorgestellt. Die Notwendigkeit eines zeitlichen und kostenseitigen Montage-Demontage-Abgleichs ist für die Erreichung der Ziele unabdingbar, da im Rahmen des Entwicklungsprozesses keine Suboptima entstehen sollen. Im Anschluss wird an einem Beispiel aus dem direkten Forschungsumfeld der Autoren, ein 48V-Submodul eines HV-Speichers, das Vorgehen und die Rückschlüsse für die Entwicklung und Konstruktion veranschaulicht. Abschließend werden die gewonnenen Erkenntnisse für die Konstruktionssystematik von HV-Speichern verallgemeinert und dienen der Beantwortung folgender Fragen: Welche Größen beeinflussen unmittelbar die Instandsetzung- und damit die Wieder- bzw. Weiterverwendbarkeit? - Welche Aspekte müssen zur Sicherstellung der Nachhaltigkeit (z.B. Langlebigkeit und Zweitnutzung der Module etc.) zwangsweise berücksichtig werden? - Wie kann sichergestellt werden, dass mit nicht formstabilen und thermisch komplexen Einzelteilen (Zellen) eine sichere Demontagefähigkeit gewährleitet ist? - Welche Detektierungsverfahren und -methoden sind notwendig?

Keywords: Konstruktionssystematik, Hochvolt-Speicher, Nachhaltigkeit, Instandsetzungsfähigkeit

\section{Stand der Technik - Modularität versus monolithische Auslegung}

Der Hochvolt-Speicher (HV-Speicher) verursacht einen Großteil der Kosten im elektrischen Antriebsstrang und bestimmt als eine wesentliche Baugruppe von Hybridfahrzeugen und Battery Electric Vehicle (BEV) die Lebensdauer und Nutzungsmöglichkeiten dieser Fahrzeuge. Ihm kommt deshalb eine besondere Rolle zum Erhalt des Nutzwertes von BEVs zu. In früheren Konzepten spielten aufgrund von sicherheitsrelevanten Auslegungen, Instandsetzungsfähigkeit und Rekonfigurierbarkeit eine untergeord- 
nete Rolle. Aktuell erfolgt aufgrund der in (Wagenhaus \& Heinicke, 2015, S. 107) genannten Gründe ein Umdenken hin zur Verwendung der HV-Speicher im Second-LifeZyklus. Eine systematische Betrachtung der nachhaltigen Gestaltung von HV-Speichern ist daher unumgänglich. Einbezogen werden dabei unteranderem die verwendeten Materialien für die Batteriezellen und Hilfskonstruktionen sowie die genutzte Zelltechnologie und -gestaltung. Darüber hinaus haben vor allem die konstruktiven Verfahren und Methoden zur Konfektionierung des HV-Speichers einen Einfluss auf dessen Nachhaltigkeit und Instandsetzungsfähigkeit. Die gewählten Gestaltungsmöglichkeiten, welche direkt von den gewählten Zelltypen abhängig sind, beeinflussen die Wieder- bzw. Weiterverwendbarkeit von Baugruppen und Komponenten. Ziel ist es, eine insgesamt hohe Nutzungsdauer des HV-Speichers zu erreichen und so den nachhaltigen Einsatz zu fördern. (Wagenhaus \& Heinicke, 2015)

Die Entwicklung von HV-Speichern ist direkt abhängig von den verfügbaren Zelltechnologien. Die Leistungsdichte (gravimetrisch und volumetrisch), die Händelbarkeit und nicht zuletzt die Zuverlässigkeit der Batteriezellen werden beim Auswahlprozess berücksichtigt. In ersten Nischenanwendungen basierten die HV-Speicher noch auf Säurebatterien bzw. sogenannten Solarbatterien (Blei-Gel-Zellen) und deren Kompaktbauweise als 6V- bzw. 12V-Block. Diese bildeten eine Baugruppe. Die aus der Bauart resultierende Reihenschaltung zur Erlangung notwendiger Betriebsspannungen führte dazu, dass solche Konzepte zwangsläufig eine einfache Austauschbarkeit von einzelnen Batterien aufwiesen (z.B. Vorläufer des Streetscooter, der Eco Carrier aus Wunsdorf).
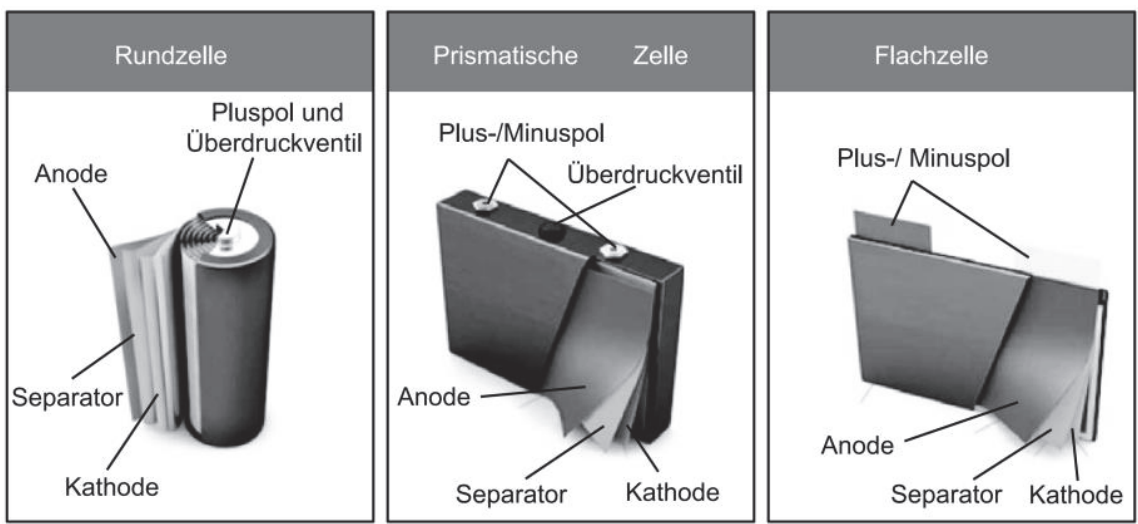

Abbildung 1: Grundlegende Batteriezelltypen (Kampker, 2014, S. 54) 


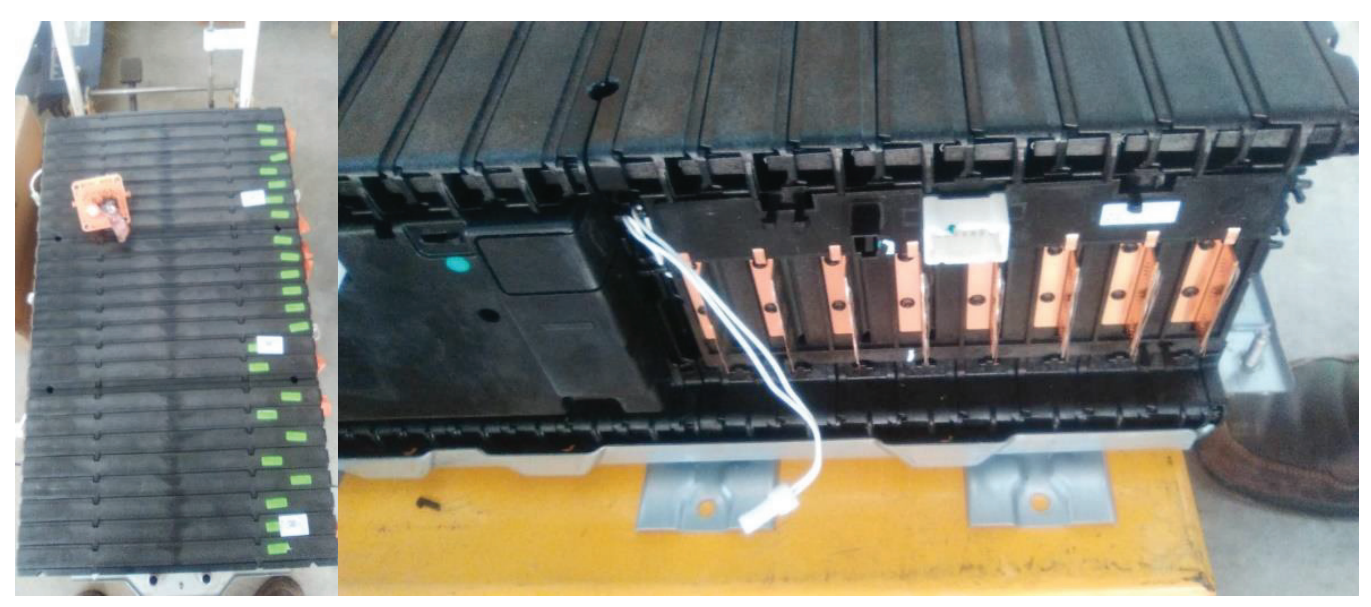

Abbildung 2: Defekter HV-Speicher (eigene Darstellung)

Die weitere Entwicklung und die zunehmenden Sicherheitsanforderungen (z. B. abgeschlossene, crashsichere Batteriegehäuse, EMV-Sicherheit, Unzugänglichkeit bzw. Berührungsschutz der HV-führenden Teile) resultierten in kleinen, handlicheren Batteriezellen (vgl. Abbildung 1). Einer der drei dargestellten Zelltypen wird zumeist als Eingangsgröße zum Aufbau des HV-Speichers gewählt. In Abhängigkeit des Zelltyps ergibt sich ein unterschiedliches, mechanisches Bestimmtheitsmaß hinsichtlich der weiteren Konzeptgestaltung. Im Einzelnen soll hier nicht auf technische Merkmale eingegangen, sondern auf (Lensch-Franzen, Gohl, Schmalz, \& Doguer, 2020) verwiesen werden.

Mit der auf Grund der steigenden Leistungsdichten zunehmenden Verbreitung der Einzelzellen (Nennspannungen je Zelle bei 2 bis 3,7V) ergaben sich neue Herausforderungen hinsichtlich der Konfektionierung von HV-Speichern. Die Entwicklung wurde früher häufig von einer monolithischen Kompaktbauweise in Kombination mit automatisierbaren Fügeverbindungen getragen. So werden bei vielen OEMs bzw. deren Zulieferern Schweißverfahren (bspw. Ultraschallschweißen) vor dem Hintergrund sicherer Verbindungen mit möglichst geringen Übergangswiderständen und einem hohen Anteil an Prozessautomatisierungen eingesetzt. Dies führt bei monolithischen Bauweisen, wie in Abbildung 2 dargestellt, im Falle des Versagens einer Batteriezelle zum Totalausfall des HV-Speichers. So folgt dem Zellschaden im dargestellten HV-Speicher eines SUVs, hervorgerufen durch einen Produktionsfehler (fehlender Wärmeleitkleber an einer Zelle) der Gesamtausfall des HV-Speichers. Der Ausfall erfolgte nach einer Nutzungs- bzw. Betriebszeit von ca. $40 \mathrm{~h}$ und stellte einen finanziellen Schaden von ca. 
$17.000 €$ (Neu-Akku) dar. Die Instandsetzung des HV-Speichers wurde durch die Nichtlösbarkeit der Zellen aus dem Verbund und darüber hinaus auch vom Gehäuse, welches durch die direkte thermische Kopplung als Zwangskühlung diente, verhindert.

Für die Instandsetzungsfähigkeit bzw. das Erschließen von Wieder- und/oder Weiterverwendungsmöglichkeiten der Baugruppen und Einzelteile kommt ein Abgleich der Montage-Demontagevorgänge in Betracht. Um den sicherheitstechnischen Anforderungen bei Montage und Demontage (z.B. Unterteilung in 48V-Submodule) zu entsprechen, gilt es, die Einflüsse der Demontierbarkeit gegen die Kostensteigerung der daraus resultierenden Montageaufwände abzuwägen.

Für den jeweiligen Anwendungsfall werden die Anforderungen an die Eigenschaften der Zellen prioritär abgearbeitet (vgl. Tabelle 1), wobei sich zeigt, dass wesentliche Einflussgrößen auf den Montage-Demontageabgleich hinter den rein technischen Anforderungen zurückgestellt werden. Dies führt zur Verlagerung von Kosten in die Montageprozesse mit der Folge einseitiger Optimierungsroutinen, welche die Suboptima der kostenoptimierten Montage- bzw. Fügeoperationen priorisieren. (Kampker, 2014)

Die zukünftige Auslegung von HV-Speichern muss deshalb neben der Modulbauweise vor allem deren Demontagefähigkeit vor dem Hintergrund der gewählten Fügeverfahren berücksichtigen, um auf diese Weise die Instandsetzungsfähigkeit und die nachhaltige Nutzung der HV-Speicher, auch im Schadensfall, zu gewährleisten.

\section{Vorstellung des Experimentierumfeldes - die instandsetzungsfähige Hochleistungsbatterie}

Im betrachteten Anwendungsfall wird ein HV-Speicher mit der Nennspannung von $400 \mathrm{~V}$ aus Submodulen mit der Nennspannung von 48V aufgebaut. Der zu realisierende Modulträger wurde vor dem Hintergrund maximaler Baufreiheit und Fahrleistungen ausgewählt, um auf diesem Weg die Maximalanforderungen an die jeweiligen Batteriezellen zu stellen. Dementsprechend führte die technisch dominierte Auswahl der Batteriezellen zum Einsatz von Flachzellen oder Pouchzellen. Diese stellen vor dem Hintergrund ihrer mechanisch/thermischen Eigenschaften, bspw. einer begrenzten mechanischen Bestimmtheit der Hülle und einer thermisch veränderbaren Kubatur in Abhängigkeit von der elektrischen Beanspruchung, die Maximalanforderungen an die konstruktive nachhaltige Gestaltung von HV-Speichern dar und sind somit als anspruchsvolle Versuchskörper zu betrachten. Die negativen Aspekte werden durch die 
hohen Energiedichten in Verbindung mit einem hohen Potential für die direkte Temperierung ausgeglichen (Verhältnis Oberfläche/Volumen). Die Eigenschaften der gewählten Pouchzelle sind in Tabelle zusammengefasst.

Tabelle 1: Eigenschaften Pouchzelle

\begin{tabular}{llll}
\hline Eigenschaft & Spezifizierung & Eigenschaft & Spezifizierung \\
\hline Kapazität & $75 \mathrm{Ah}$ & Masse & max. 1.780g \\
$\begin{array}{l}\text { Gravimetrische } \\
\text { Energiedichte }\end{array}$ & $156 \mathrm{Wh} / \mathrm{kg}$ & Zelldimension & $268 \times 265 \times 13 \mathrm{~mm}$ \\
$\begin{array}{l}\text { Volumetrische } \\
\text { Energiedichte }\end{array}$ & $301 \mathrm{Wh} / \mathrm{L}$ & Nennspannung & $3,7 \mathrm{~V}$ \\
$\begin{array}{l}\text { Gravimetrische } \\
\text { Energiedichte }\end{array}$ & $156 \mathrm{Wh} / \mathrm{kg}$ & Entladestrom & (cont./peak) \\
Impedanz & max. $0,55 \mathrm{~m} \Omega$ & Ladestrom & $600 \mathrm{~A} / 1125 \mathrm{~A}$ \\
\hline
\end{tabular}

Die Gesamtkonzeption von HV-Speicher und Submodulen erfolgt vor dem Hintergrund der Zugänglichkeit und Separierbarkeit der jeweiligen Submodule im Gesamtsystem sowie der Absicherung der Demontagefähigkeit der Module. Dies dient dazu, die damit verbundenen Einflüsse auf die Montageoperationen gezielt darzustellen und herauszuarbeiten. Alle Verbindungselemente wurden demontageorientiert, im Erstentwurf größtenteils mittels Handmontage, ausgelegt. Technische Anforderungen an z. B. Polfahnenverbinder wurden entsprechend den notwendigen Flächenpressungen mittels Hilfskonstruktionen derart gestaltet, dass auch hier die Demontagefähigkeit gewährleistet ist.

Aus der technischen Spezifizierung der Zelle ergeben sich erhöhte Anforderungen an die Modulbauweise hinsichtlich konstruktiver Auslegung und Auswahl der Montageoperationen (Hauptzeiten, welche direkt kostenbestimmend sind) sowie der dazu notwendigen Handlingoperationen (Nebenzeiten). Im Allgemeinen gilt auch hier, dass der Wertschöpfungsanteil (Montagezeiten in Anzahl und Umfang) minimiert werden soll. Dazu kommt die konsequente Eliminierung der Nebenzeiten, insbesondere, wenn diese ablaufbestimmend werden. 


\section{Anwendung der Konstruktionssystematik am Beispiel}

Das Vorgehen im Rahmen der nachhaltigen Konstruktion von HV-Speichern wird an einer exemplarischen Baugruppe des 48V-Submoduls aus dem Forschungsumfeld der Autoren vorgestellt.

Aus den finanziellen Zusammenhängen in der VDI 2243 (VDI, Verein Deutscher Ingenieure, 2002) zur Materialkreislaufeignung bzw. Komponentenkreislaufeignung wird die Formel betreffend der Komponentenweiter- bzw. Wiederverarbeitung herangezogen:

$$
K E_{x}=\frac{\text { Kosten Neuteil (Batteriezelle) }+ \text { Beseitigungskosten in Euro }}{\text { Recycling }- \text { Kosten Altteil in Euro }}
$$

Im Anwendungsfall sind die Bezugskosten für die Zellbeschaffung zumeist nicht beeinflussbar (abhängig von den Materialpreisen und den Fertigungstechnologien). Für die Beseitigungskosten ist davon auszugehen, dass diese nicht zuletzt durch den spezifischen Aufbau der Zellen (z.B. Brandgefahr, Verwendung giftiger Stoffe, etc.) und begrenzte Entsorgungsmöglichkeiten durchschnittlich hoch sind und nicht kurzfristig verringert werden können. Beide Kostenarten hängen damit von der Zelltechnologie ab und können somit nicht unmittelbar beeinflusst werden.

Die Recyclingkosten subsumieren die mechanischen Demontagekosten (abhängig von Anzahl und Ausführungszeit sowohl für Modulentnahme aus Gesamtsystem und der Zellentnahme aus Modul), die Aufarbeitungskosten (Detektieren des fehlerhaften Bauteils bezüglich Zelle/Modul) und die Logistikkosten (Transport zur Instandsetzungseinheit, Rücktransport zum Fahrzeug in Abhängigkeit vom Transportgut (Pack, Modul, Zelle)).

Für den Montage-Demontageabgleich gilt also, dass das Optimum aus kostenminimierenden Montageaufwendungen bei gleichzeitiger Sicherstellung grundlegender Demontagefähigkeit bei vertretbarem Aufwand und Materialverlust gefunden werden muss. Im vorliegenden Anwendungsfall wurden zur Erstellung eines systematischen Vorgehens auf den Entwicklungsprozess des HV-Speichers zwei Sichtweisen im sequentiellen Abgleich verfolgt, um so mit dem Entwicklungs- und Konstruktionsfortschritt jeweils die Demontage abzusichern. Hierzu wird im ersten Schritt die grundlegende Struktur des HV-Speichers vor dem Hintergrund der Minimierung anfallender Kosten festgelegt. Demontiert werden kann das Batteriepack an sich, ein oder mehrere Module sowie eine oder mehrere Zellen innerhalb eines Moduls. Die Entnahme bzw. der Rückbau kompletter Akkupakete führt zu einem Transportgut mit sehr hohem 
Gewicht und nicht zuletzt einem Gefahrgut. Gewicht, Zugänglichkeit und grundlegende Demontageeignung sprechen hier für die Entnahme eines Moduls. Dem Demontagevorgang auf Zellebene stehen große Sicherheitsbedenken entgegen (Eingriffe an stromführenden Baugruppen über einem definierten Spannungsniveau). Die Modulentnahme und der weitere Umgang mit einer Baugruppe müssen daher klar definiert sein.

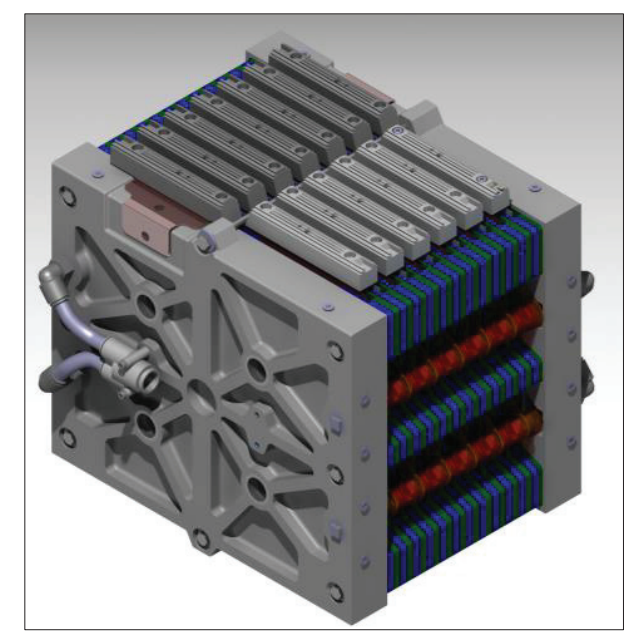

Abbildung 3: 48V-Submodul (eigene Darstellung)

Das entwickelte Modul (vgl. Abbildung 3) wird im Weiteren kurz erläutert. Die formulierten Randbedingungen an das Gesamtsystem gehen in die Top-Down-Systemauslegung ein und sind hauptsächlich verantwortlich für das Ergebnis der Zellauswahl. Damit bilden die in oben vorgestellte Pouchzelle (prozessseitig bedingt geeignet), die aufgrund der Anforderungen an die Zelltechnik (späteres Fahrprofil etc.) gewählt wurde, sowie einige Randbedingungen, die das Modul erfüllen soll, die Grundlage des Entwicklungsprozesses. Dazu gehören unter anderem die thermische Regulierbarkeit, die Erfüllung von Sicherheitsaspekten und nicht zuletzt die zerstörungsfreie Demontage und Instandsetzbarkeit. Zur Erfüllung dieser Randbedingungen und des korrekten Handlings der Pouchzellen wurde ein Sub-Modul entwickelt, dass sich unter anderem durch eine innenliegende Spannvorrichtung, eine lösbare Klemmverbindung der Polfahnen und fluiddurchströmte Mikrokanalkunststofffolien auszeichnet. Die Montagegrobstruktur des Moduls ist in Abbildung 4 dargestellt und verdeutlicht bereits einige Aspekte des grundlegenden Vorgehens im späteren Montage-Demontageabgleich. 
Im Entwicklungsprozess wird in gewissem Maße bereits bei der Zellauswahl, also in frühen Stadien der Funktionalen Entwicklung, der Anteil der zerstörungsfreien Einzelteile festgelegt. Diese Einzelteile, welche rückgewinnungswürdig sind und damit im Schadensfall oder am Ende des First-Life-Zyklus einem neuen System hinzugefügt werden können, bestimmen nun sowohl die verwendete Fertigungs- bzw. Fügetechnologie und als logischen Abgleich mit dessen Eigenschaften die notwendigen Hilfskonstruktionen. Anhand der Stückliste werden rückgewinnungsfähige Einzelteile/Baugruppen bestimmt und setzen dementsprechend Marker im Sinne der Wieder- bzw. Weiterverwendung prioritär zu den zu gestaltenden Produktumfängen.

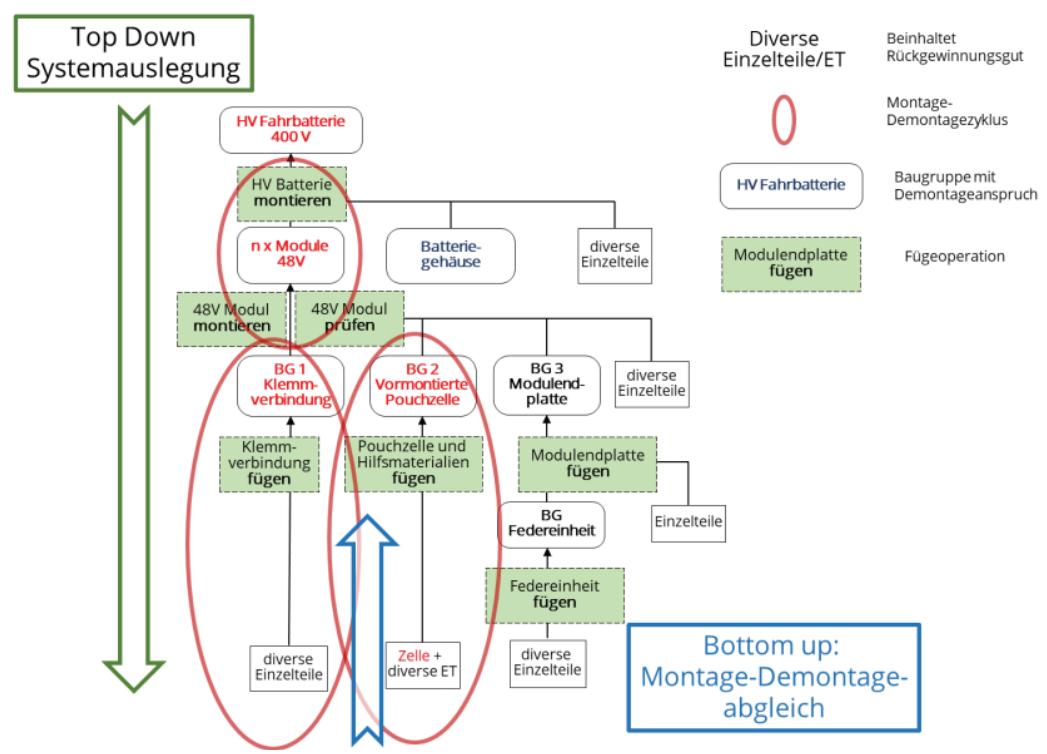

Abbildung 4: Montagegrobstrukturierung des HV-Speichers (eigene Darstellung)

Im nächsten Schritt müssen die daraus resultierenden rückgewinnungswürdigen Einzelteile und darauf aufbauende Baugruppen bestimmt werden, auf welche im Konstruktionsprozess der Fokus gelegt wird.

Entscheidend für die aufwandsminimierte Rückgewinnung der Zelle sind im Beispiel zum einen die Baugruppe BG2 „Vormontierte Pouchzelle" und zum anderen die Baugruppe BG1 „Klemmverbindung“, welche anhand des aufgebauten Schemas (vgl. Abbildung 5) näher betrachtet wird. Die Darstellung basiert auf dem Innovationsprozess nach Cooper und wurde für die beschriebene Problemstellung angepasst (Cooper, 
1983). Es stehen hierbei zwar die konstruktive Gestaltung der Baugruppen, aber vielmehr die Fügeverfahren im Vordergrund, da diese im Rahmen der Optimierungsroutine des Konstruktionsprozesses den Gradmesser für den Montage-Demontageabgleich bilden.

Da es eine direkte Rückkopplung zwischen der Konzeptlösung des Fügens und der damit verbundenen Möglichkeit der Rückgewinnung gibt, gilt es grundsätzlich, jede Gestaltungslösung vor dem Hintergrund der Demontagefähigkeit abzuprüfen. Die gewählten Anforderungen an die Demontage werden in Anlehnung an die VDI 2243 gewählt.

Aus der Montage- und der Demontageabschätzung entsteht vor dem Hintergrund der wechselseitigen Beeinflussung ein ausbalanciertes Lösungskonzept, dass in Abhängigkeit von den Eingangsbedingen die Einzelteile zielorientiert auslegt.

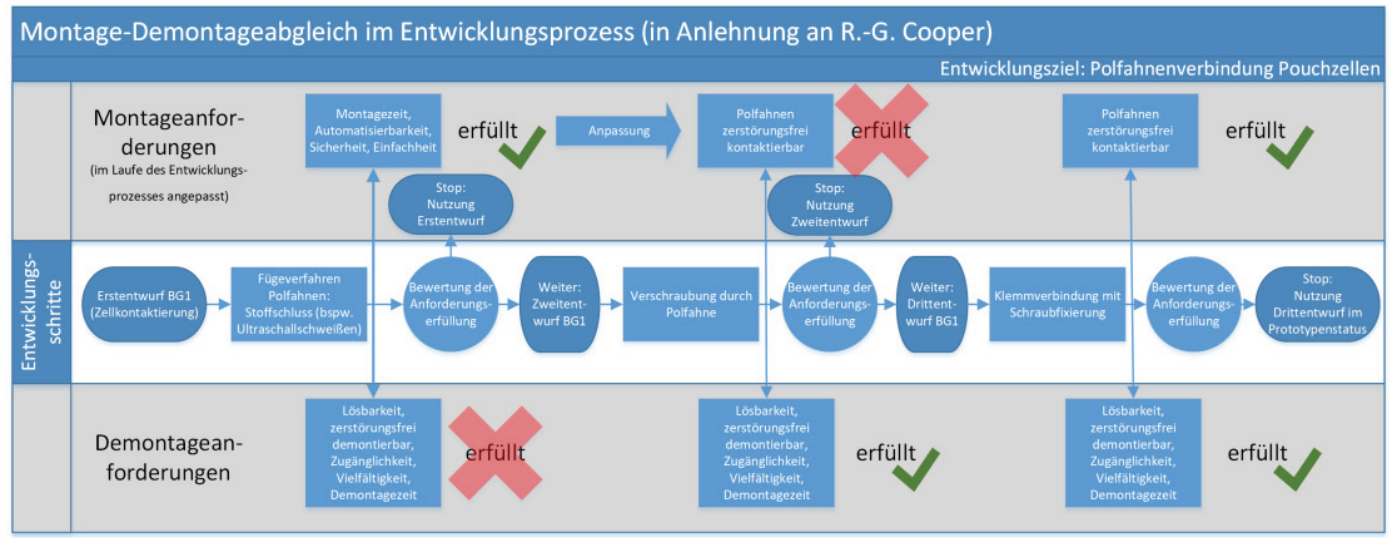

Abbildung 5: Feinauslegung innerhalb der nachhaltigen Konstruktion von HV-Speichern (eigene Darstellung)

Je nach prioritärer Zielsetzung können damit montageseitige, hier zumeist kostenseitige Ansätze verfolgt werden, ohne die grundsätzliche Wieder- und Weiterverwendungsmöglichkeit zu unterlaufen. Gleichzeitig ermöglicht das Vorgehen unter Berücksichtigung der VDI 2243 Rückschlüsse auf die Eignung eingesetzter Materialen vor dem Hintergrund der Weiter- bzw. Wiederverwendbarkeit. 


\section{Fazit}

Mit der vorgelegten Methode ist es den Entwicklern von HV-Speichern möglich, von der ersten Idee über die systemische Auswahl der Komponenten bis hin zur technischen Gesamt- und Einzelteilauslegung eine konsequente Orientierung auf nachhaltigen Mitteleinsatz vorzunehmen. Besonderer Wert kommt dabei der systemischen Abprüfung entlang des gesamten Entwicklungsprozesses zu, gepaart mit der Prioritätensetzung auf kritische Komponenten. Gleichzeitig erfolgt im engeren Sinne durch den Montage-Demontageabgleich eine systematische Rückkopplung der im Prozess gewonnenen Ergebnisse auf die verwendeten Einsatzmaterialen, ihre Gestaltung und vor allem hinsichtlich ihrer Prozessfähigkeit für die Montage- und Demontageprozesse.

Die aktuell verwendeten Batteriezellen ziehen insbesondere bei Hochleistungszellen in späteren Entwicklungsstufen einen hohen Entwicklungsaufwand nach sich. Dies spiegelt sich häufig in einem hohen Komplexitätsgrad wieder, welcher sich negativ auf die entstehenden Kosten auswirkt. Am vorliegenden Beispiel ist ersichtlich, dass ohne eine Berücksichtigung prozessseitiger Eignung (Montage/Demontage) bzw. Gestaltung der Einsatzmaterialen, der HV-Speicher nur begrenzt ökonomisch optimiert werden kann. Der vorliegende Fall zeigt auf, wie der Entwicklungsprozess der Baugruppen im HV-Speicher unter Berücksichtigung des Nachhaltigkeitsaspektes systematisch erschlossen werden kann.

Im Zuge der laufenden Betrachtung erscheint es von besonderer Bedeutung, dass die Entwicklungsprozesse insgesamt besser abgestimmt sein müssen. Ein führender EFahrzeughersteller geht konsequent den Weg der eignen Zellentwicklung und -produktion, welches vor dem Hintergrund der hier vorliegenden Untersuchungen dringend notwendig wird. Alternative Formen der gemeinsamen lieferkettenübergreifenden Entwicklung stellen hier ebenfalls praktikable Wege dar. Für die in den Untersuchungen beschriebenen Hochleistungszellen sehen die Autoren insbesondere wegen der nur begrenzten Formstabilität und den erhöhten Anforderungen an das Handling im Produktionssystem nur begrenzte Entwicklungsmöglichkeiten für die Massenproduktion, wenn es nicht gelingt, die hohen Aufwendungen aus der Akkukonfektionierung teilweise durch Veränderung der Batteriezelleigenschaften zu kompensieren. 


\section{Danksagung}

Die Autoren danken dem Europäischen Fonds für regionale Entwicklung und dem Land Sachsen-Anhalt, die das Projektvorhaben KeM, in dessen Rahmen diese Arbeit entstand, fördern.

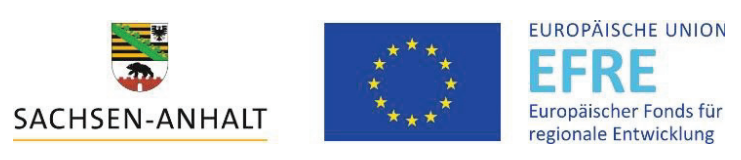

\section{Literaturverzeichnis}

Cooper, R. G. (Februar 1983). A process model for industrial new product development.

IEEE Transactions on Engineering Management, S. 2-11.

Kampker, P. D.-I. (2014). Elektromobilproduktion. Aachen: Springer-Verlag Berlin Heidelberg.

Lensch-Franzen, C., Gohl, M., Schmalz, M., \& Doguer, T. (09. Oktober 2020). Von der Zelle zum Batteriesystem -

Verschiedene Zellformate und ihre Systemintegration. MTZ - Motortechnische Zeitschrift, S. 72-75.

VDI, Verein Deutscher Ingenieure. (Juli 2002). VDI 2243 - Recyclingorientierte Produktentwicklung.

Wagenhaus, G., \& Heinicke, M. (April 2015). Sustainability in the car-based mobility: The case of the electric vehicle Editha.

International Journal of Energy Sector Management, S. 105-119.

\section{Kontakt}

Robert Kretschmann, M. Sc.,

Prof. Dr.-Ing. Christiane Beyer

Otto-von-Guericke Universität Magdeburg

Institut für Maschinenkonstruktion

Lehrstuhl Produktentwicklung und Konstruktion

Universitätsplatz 2

39106 Magdeburg

https://lpk.ovgu.de/

Oberingenieur Gerd Wagenhaus

Otto-von-Guericke Universität Magdeburg

Institut für Arbeitswissenschaft, Fabrikautomatisierung und Fabrikbetrieb

Universitätsplatz 2

39106 Magdeburg

https://www.iaf.ovgu.de/ 